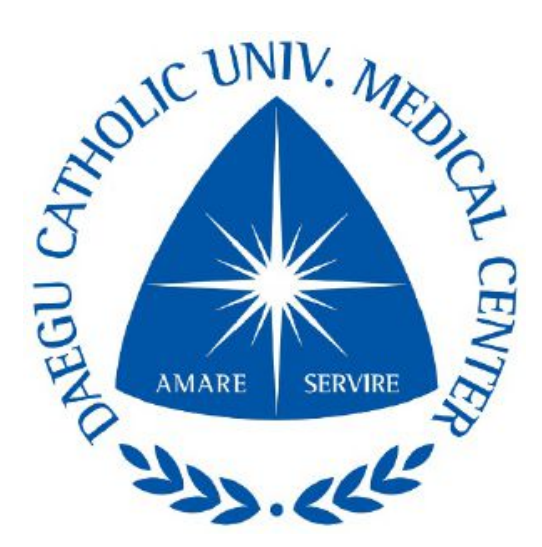

\title{
The Effect of Group Art Therapy and the Group Psychoeducation in Adolescents With Internet and Smartphone Overuse
}

${ }^{1}$ Department of Psychiatry, Catholic University of Daegu School of Medicine, Daegu, Republic of Korea ${ }^{2}$ Department of Psychiatry, Kyungpook National University School of Medicine, Daegu, Republic of Korea

\section{Objectives}

The objective of this study was to evaluate therapeutic effectiveness of group art therapy and group psychoeducation program on adolescents' internet or smartphone overuse.

\section{Background and aims}

There are no standardized guidelines for the treatment of internet or smartphone overuse. Previous studies have shown that group art therapy has a positive effect on mental health issues, including depression, anxiety, and addiction. We assessed whether group art therapy improves co-morbid psychiatric symptoms in adolescents with internet or smartphone overuse.

Table 1. Sociodemographic characteristics of the study population

\begin{tabular}{lcc}
\hline & $\begin{array}{c}\text { Group } \\
\text { art therapy } \\
(\mathrm{n}=11)\end{array}$ & $\begin{array}{c}\text { Group } \\
\text { psychoeducation } \\
(\mathrm{n}=12)\end{array}$ \\
\hline Age (mean \pm SD) & $12.55 \pm 0.934$ & $10.49 \pm 0.900$ \\
Sex & $4(36.4)$ & $5(41.7)$ \\
Male & $7(63.6)$ & $7(58.3)$ \\
Female & & \\
Parent marital status & $8(72.7)$ & $10(83.3)$ \\
Married & $2(18.2)$ & $2(16.7)$ \\
Divorced & $1(9.1)$ & $0(0.0)$ \\
Widowed & $3(27.3)$ & $6(50.0)$ \\
School performance & $5(45.5)$ & $5(41.7)$ \\
High & $3(27.3)$ & $1(8.3)$ \\
Middle & $2(18.2)$ & $1(8.3)$ \\
Low & $9(81.8)$ & $11(91.7)$ \\
History of mental disorders & $14.84 \pm 2.286$ & $13.82 \pm 8.162$ \\
Yes & & \\
No & $9.08 \pm 8.777$ & $11.84 \pm 10.607$ \\
Time on smartphone use/w & & \\
(mean \pm SD) & & $5(41.7)$ \\
Time on internet game use/w & & $7(58.3)$ \\
(mean \pm SD) & & \\
Internet addiction & $7(63.6)$ & $2(16.7)$ \\
Addicted \& High risk group & $4(36.4)$ & $10(83.3)$ \\
Normal group & & \\
Smartphone addiction & $4(36.4)$ & \\
Addicted \& High risk group & $7(63.6)$ & \\
Normal group & & \\
\hline SD $:$ stand & & \\
\hline
\end{tabular}

SD : standard deviation, $\mathrm{w}:$ week

\section{Materials and methods}

Twenty-three elementary and middle-school students participated in this study. All participants were administered clinical scales before and after the intervention. The Korean version of Young's Internet Addiction Test (YIAT), the Korean Smartphone Addiction Proneness Scale (SAPS), the Korean version of the Children's Depression Inventory (CDI) and the State/Trait Anxiety Inventory for Children (SAIC, TAIC) were used .Among the participants, 11 students were assigned to participate in group art therapy, and the other 12 students were assigned to participate in group psychoeducation. Both interventions were conducted by psychiatrists and psychologists in
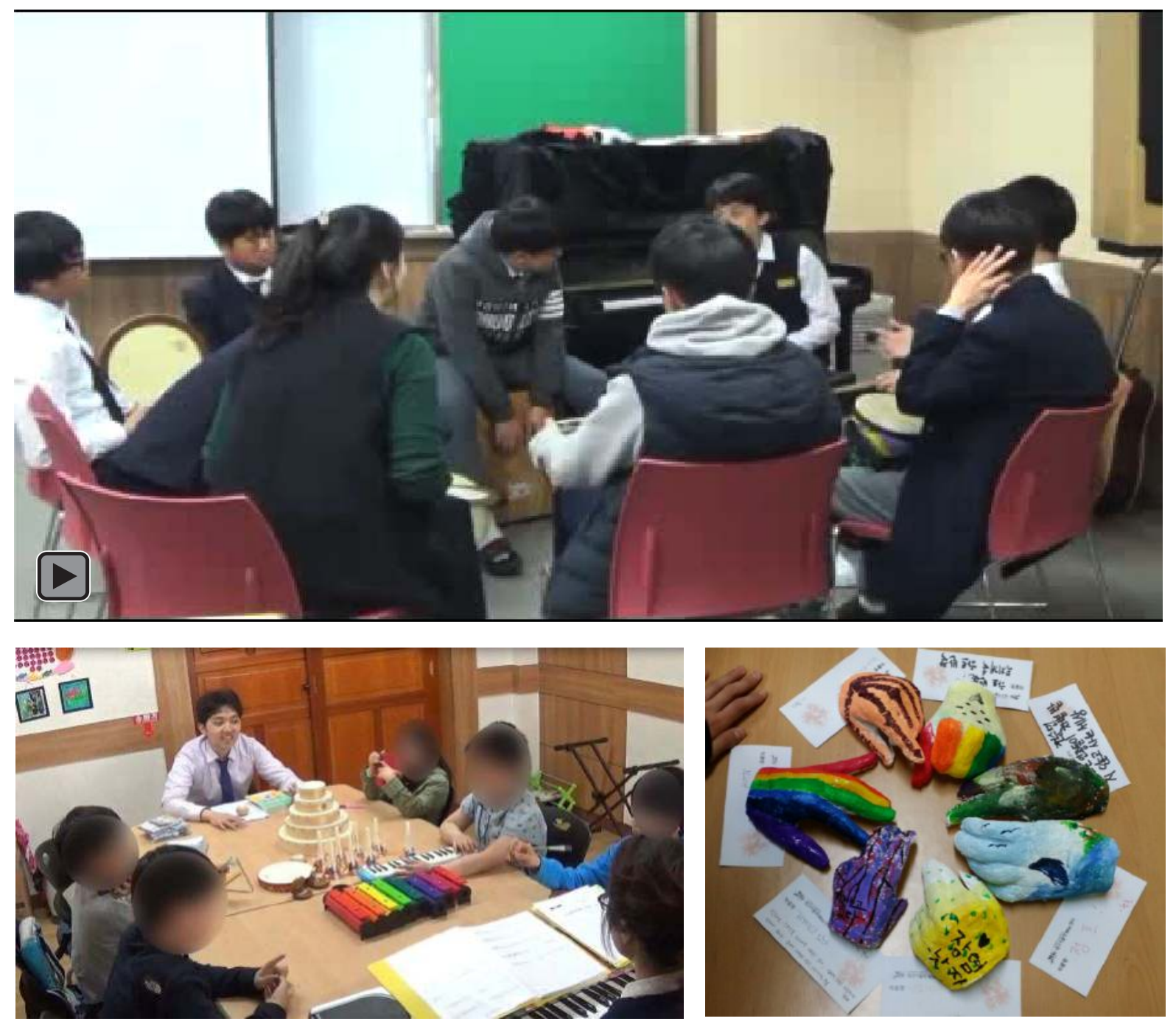

Results

There was significant decrease in total scores of SAPS $(p=0.045)$, CDI $(p=0.003)$ and SAIC $(p=0.009)$ after performing group art therapy. After performing group psychoeducation, only total scores of YIAT $(p=0.013)$ and SAPS $(p=0.003)$ decreased significantly

Table 2. Differences in psychometric test scores between before and after gro up art therapy

\begin{tabular}{lcccc} 
& \multirow{2}{*}{ Before } & \multirow{2}{*}{ After } & \multicolumn{2}{c}{$\begin{array}{c}\text { Wilcoxon signed } \\
\text { rank test }\end{array}$} \\
\cline { 2 - 5 } & Mean $\pm \mathrm{SD}$ & Mean $\pm \mathrm{SD}$ & $\mathrm{Z}$ & $\mathrm{P}$ \\
\hline YIAT & $41.55 \pm 17.172$ & $39.09 \pm 17.196$ & -.513 & $0.045^{*}$ \\
SAPS & $39.45 \pm 4.083$ & $35.00 \pm 6.943$ & -2.002 & 0.608 \\
CDI & $20.36 \pm 9.341$ & $8.09 \pm 7.905$ & -2.938 & $0.003^{*}$ \\
TAIC & $43.09 \pm 6.670$ & $40.00 \pm 9.930$ & -1.252 & 0.211 \\
SAIC & $38.91 \pm 6.204$ & $31.09 \pm 7.063$ & -2.627 & $0.009^{*}$ \\
\hline
\end{tabular}

$*: \mathrm{p}<0.05, \mathrm{SD}:$ Standard deviation, YIAT : Young's Internet Addiction Test, SAPS : Korean Sm artphone Addiction Proneness Scale, CDI : Children depression inventory, SAIC : State Anxiety Inventory for Children, TAIC : Trait Anxiety Inventory for children

Table 3. Differences in psychometric test scores between before and after gro up psychoeducation

\begin{tabular}{lcccc} 
& \multicolumn{2}{c}{ Before } & After & \multicolumn{2}{c}{$\begin{array}{c}\text { Wilcoxon signed } \\
\text { rank test }\end{array}$} \\
\cline { 2 - 5 } & Mean $\pm \mathrm{SD}$ & Mean $\pm \mathrm{SD}$ & $\mathrm{Z}$ & $\mathrm{p}$ \\
\hline YIAT & $37.00 \pm 12.358$ & $26.33 \pm 6.998$ & -2.473 & $0.013^{*}$ \\
SAPS & $33.58 \pm 4.660$ & $26.17 \pm 6.408$ & -2.986 & $0.003^{*}$ \\
CDI & $4.42 \pm 4.358$ & $3.92 \pm 7.902$ & -1.207 & 0.227 \\
TAIC & $26.42 \pm 6.374$ & $25.83 \pm 5.859$ & -.102 & 0.919 \\
SAIC & $26.50 \pm 5.368$ & $25.67 \pm 7.426$ & -.891 & 0.373 \\
\hline
\end{tabular}

* : $\mathrm{p}<0.05$, SD : Standard deviation, YIAT : Young's Internet Addiction Test, SAPS : Korean Sm artphone Addiction Proneness Scale, CDI : Children depression inventory, SAIC : State Anxiety Inventory for Children, TAIC : Trait Anxiety Inventory for children

\section{Conclusions}

Both interventions showed improvement in adolescent's addictive behaviors. Group art therapy was especially effective in decreasing co-morbid depressive and anxiety symptoms. Group art therapy can thus be used to treat adolescents with internet or smartphone overuse and co-morbid mental health symptoms. 